\title{
Interruption of breast cancer care and importance of inter-hospital cooperation during the COVID-19 pandemic: A case report of advanced breast cancer in Fukushima, Japan
}

\author{
Akihiko Ozaki $^{1}$, Yudai Kaneda ${ }^{2}$, Yuki Senoo ${ }^{1}$, Masahiro Wada ${ }^{1}$, Tomohiro Kurokawa ${ }^{1}$, \\ Toyoaki Sawano ${ }^{1}$, Masaharu Tsubokura ${ }^{3}$, Tetsuya Tanimoto ${ }^{1}$, Yoshiaki Kanemoto ${ }^{1}$, \\ Tomozo Ejiri $^{1}$, H Shimmura ${ }^{1}$, and Norio Kanzaki ${ }^{1}$ \\ ${ }^{1}$ Jyoban Hospital of Tokiwa Foundation \\ ${ }^{2}$ Hokkaido University \\ ${ }^{3}$ Fukushima Medical University School of Medicine
}

February 8, 2022

\begin{abstract}
We experienced the case of a patient with advanced breast cancer who failed to receive comprehensive care despite regular video conferencing with her physician during the COVID-19 pandemic, resulting in delayed detection of liver metastasis. Inter-hospital collaboration is required to provide uninterrupted cancer care to those disproportionately affected by crises.
\end{abstract}

Interruption of breast cancer care and importance of inter-hospital cooperation during the COVID-19 pandemic: A case report of advanced breast cancer in Fukushima, Japan

Authors:

Ozaki $\mathrm{A}^{1,2^{*}}$, Kaneda $\mathrm{Y}^{3}$, Senoo $\mathrm{Y}^{1}$, Wada $\mathrm{M}^{1,4}$, Kurokawa $\mathrm{T}^{5}$, Sawano $\mathrm{T}^{5,6}$, Tsubokura $\mathrm{M}^{6}$, Tanimoto $\mathrm{T}^{7}$, Kanemoto $\mathrm{Y}^{5}$, Ejiri $\mathrm{T}^{5}$, Shimura $\mathrm{H}^{8}$, and Kanzaki $\mathrm{N}^{5}$

Affiliation s:

1. Department of Breast Surgery, Jyoban Hospital of Tokiwa Foundation, Iwaki, Fukushima, Japan

2. Department of Gastrointestinal Tract Surgery, Fukushima Medical University, Fukushima, Japan

3. School of Medicine, Hokkaido University, Sapporo, Hokkaido, Japan

4. Department of Breast Surgery, Sano Kosei General Hospital, Sano, Tochigi, Japan

5. Department of Surgery, Jyoban Hospital of Tokiwa Foundation, Iwaki, Fukushima, Japan

6. Department of Radiation Health Management, Fukushima Medical University School of Medicine, Iwaki, Fukushima, Japan

7. Department of Internal Medicine, Jyoban Hospital of Tokiwa Foundation, Iwaki, Fukushima, Japan

8. Department of Urology, Jyoban Hospital of Tokiwa Foundation, Iwaki, Fukushima, Japan

\section{Corresponding author:}


Ozaki A, Department of Breast Surgery, Jyoban Hospital of Tokiwa Foundation, 57 Kaminodai, JyobanKamiyunaga-Yamachi, Iwaki, Fukushima, Japan, Tel: 81-244-22-3181; Fax: 81-244-22 - 8853; Email address: ozakiakihiko@gmail.com.

\title{
Key clinical message
}

Few case-based analyses have been conducted regarding the breast cancer patients who are disproportionately affected by the COVID-19 pandemic. This case shows that patients with metastatic disease travelling from remote areas to see their physicians may need specific support so that they can receive uninterrupted care during the pandemic.

\begin{abstract}
We experienced the case of a patient with advanced breast cancer who failed to receive comprehensive care despite regular video conferencing with her physician during the COVID-19 pandemic, resulting in delayed detection of liver metastasis. Inter-hospital collaboration is required to provide uninterrupted cancer care to those disproportionately affected by crises.
\end{abstract}

\section{Keywords}

Accessibility of health services; Breast neoplasms; Consultation; COVID-19; Referral; Telemedicine

\section{Abbreviations}

COVID-19: Coronavirus Disease 2019; CT: Computed Tomography.

\section{Introduction}

Patients with breast cancer should undergo regular follow-up throughout the course of their treatment. However, the novel coronavirus disease 2019 (COVID-19) pandemic has readily hampered the continuation of breast cancer care in many parts of the world and Japan is no exception. In Japan, one of the first cases of COVID-19 was detected in March 2020 in Tokyo, and from April to May 2020, the Japanese government declared a state of emergency in Tokyo and some other prefectures as the daily number of COVID-19 cases increased exponentially. Consequently, the majority of the hospitals restricted out-patient visits as an infection control measure against COVID-19. ${ }^{1}$ In addition, some patients refrained from visiting hospitals to avoid contracting the virus. ${ }^{2}$ However, there is not enough information regarding breast cancer patients who are disproportionately affected by the COVID-19 pandemic in terms of continuation of care.

Herein, we present the case of a patient with advanced breast cancer who resided in a remote area and could not undergo in-person follow-up. Despite undergoing remote video consultations routinely, she did not receive comprehensive breast cancer care during the COVID-19 pandemic, which resulted in delayed detection of liver metastasis.

\section{Case Presentation}

In January 2016, a 38-year-old premenopausal woman was diagnosed with right-sided breast cancer at a medical institution in her home town, Iwaki City, Fukushima prefecture, Japan. As she preferred receiving care in an urban setting, she was referred to a university hospital in Tokyo, Japan, which is approximately $200 \mathrm{~km}$ away from Iwaki City in February 2016. After extensive examinations, she was diagnosed with hormone receptor-positive (estrogen and progesterone receptor-positive [both 3b]), human epidermal growth factor receptor 2-negative $(2+$ and dual color in situ hybridization equivocal) clinical T2N1M1 Stage IV breast cancer with asymptomatic multiple metastatic bone disease (right and left ilium, thoracic vertebras 4 and 7 , and lumber vertebra 4) and symptomatic metastatic disease at a sternum.

In March 2016, endocrine treatment with tamoxifen, goserelin, and denosumab was initiated; however, it was switched to exemestane, goserelin, and denosumab in June 2017, after the computed tomography (CT) and breast ultrasonography showed an enlargement of the breast tumour and deterioration of bone metastases. Because local control could not be achieved using endocrine therapy alone, mastectomy and 
axillary dissection were performed in June 2019. The cancer subtype determined after the pathological analysis of surgical specimen was the same as that determined from the core needle biopsy performed for the initial diagnosis (estrogen and progesterone receptor-positive [3b and 3a], human epidermal growth factor receptor 2-negative [1+], Ki67 18\%), and the same medical regimen was continued thereafter. In April 2020, she underwent a CT scan and the results revealed no signs of recurrence. Her last in-person visit to the university hospital was at the end of May 2020, when she received long-acting goserelin (effective for three months). Even though the Japanese government lifted the state of emergency by the end of May 2020, she refrained from visiting the university hospital in Tokyo from Fukushima due to the persistent epidemic of COVID-19 in Japan. Thereafter, her physician provided remote video consultations, namely in July and November 2020. Exemestane was prescribed virtually, and it was confirmed that her menses had not resumed. At this stage, her physician considered that a change in the prescribed regimen was not required because of the lack of evidence indicating disease progression and the difficulty in performing extensive follow-up which is mandatory after starting a new treatment. She and her physician had been looking for a medical institution near her home where she could undergo follow-up imaging regularly. However, they had been unable to find a suitable hospital for six months.

In November 2020, the patient was referred to our hospital. She had mild symptoms with an Eastern Cooperative Oncology Group Performance Status score of 1 just before visiting our hospital. Cancer antigen 15-3 and carcinoembryonic antigen levels were elevated to $87.2 \mathrm{U} / \mathrm{ml}$ and $7.6 \mathrm{ng} / \mathrm{mL}$, respectively, and subsequent positron emission tomography in January 2021 revealed multiple liver metastases that were not detected in the examination by her previous doctor nine months ago. Even though cyclin-dependent kinase $4 / 6$ inhibitors were available in Japan at the time, instead of these agents, chemotherapy with paclitaxel, bevacizumab and denosumab was initiated in January 2021, because we assumed that her disease condition had been rapidly worsening. Follow-up imaging performed in October 2021 revealed that liver metastases had shrunk in size.

\section{Discussion}

This case highlights the factors due to which the COVID-19 pandemic may impact the breast cancer patients disproportionately. These include residing in a remote area (or travelling a long distance to their physicians) and the presence of progressive disease. In general, postoperative surveillance for early-stage breast cancer patients includes annual mammography and other adjunctive investigations, such as ultrasonography and $\mathrm{CT}{ }^{3}$ On the other hand, for metastatic breast cancer patients or those experiencing disease recurrence, investigation of serum tumor marker levels and other imaging examinations performed every few months are recommended, depending on the patients' conditions. ${ }^{4}$ Regular follow-up was particularly difficult for our patient because of the long-distance travel required for each follow-up visit and the fear of potentially contracting and spreading the infection. Indeed, travelling across prefectures was discouraged during the COVID-19 pandemic in Japan.

In our case, considering the patient's condition, her primary physician understood the importance of performing a CT scan immediately, and the patient also made an effort to find a nearby hospital. However, it eventually took seven months until she could visit our hospital, probably due to the increased burden on medical institutions brought on by the pandemic and their subsequent reluctance to accept new patients. Another reason could be a scarcity of specialist physicians and hospitals specializing in providing care to cancer patients in rural areas such as Fukushima. While the collaboration between medical institutions is well established, both in medical communities and society at large, its importance has been reiterated during the COVID-19 pandemic. It has become increasingly difficult to find a hospital to refer patients to now that many hospitals are overburdened due to the pandemic and restricting the acceptance of new patients as a countermeasure. If our patient or her primary physician could have found a medical institution earlier, metastasis might have been detected in time.

This case also demonstrates the limitations of remote video consultations for cancer during the COVID-19 pandemic. In fact, there has been a rapid increase in remote video consultations for patients with various diseases, including cancer. ${ }^{5}$ Similar to our case, some hospitals have started to practice telemedicine and 
providing drug prescriptions virtually to patients who cannot visit hospitals for various reasons during the COVID-19, which was not widely practiced in Japan prior to the pandemic. ${ }^{6}$ However, without finding a nearby collaborating hospital, where the necessary examinations can be performed and treatments can be received when required, telemedicine should not be thoughtlessly implemented as a major strategy for provision of care, especially for patients with metastatic cancer or progressive disease. ${ }^{7}$

Considering that the COVID-19 pandemic is occurring as successive waves in Japan, it is necessary to not only rely on the introduction of novel telemedicine technology but also to reform and adjust the traditional referral system for medical institutions, especially between urban and rural areas, so that it is suitable for the post-pandemic era. Telemedicine can be a useful option; however, patients who have been receiving treatment at hospitals in urban areas should be able to continue their treatment in rural areas even when it becomes difficult for them to visit hospitals in urban areas. ${ }^{8}$

Therefore, it is important for medical institutions to take this opportunity to recognize the advantages and disadvantages of telemedicine. In general, remote video consultation is an effective method for patients with skin diseases that can be detected visually, or for follow-up of patients with stable chronic conditions, such as hypertension. ${ }^{9}$ However, in cases like ours, the indication of telemedicine for patients requiring specialized diagnostic imaging on a regular basis is currently limited, and it is important to understand its limitations before selecting suitable patients.

\section{Conclusions}

In conclusion, we reported the case of a patient with advanced breast cancer who failed to receive comprehensive care despite regular video conferencing with her physician during the COVID-19 pandemic, resulting in delayed detection of liver metastasis. Therefore, inter-hospital collaboration is required to provide uninterrupted cancer care to patients who are disproportionately affected by crises, and such collaborations are crucial for safe and efficient practice of telemedicine for cancer patients during the COVID-19 pandemic.

\section{Acknowledgements:}

We express our gratitude to Editage for its extensive revision of the manuscript and English proofreading.

\section{Author contributions:}

Ozaki A, Kaneda Y and Senoo Y drafted the manuscript. All authors made critical revisions of the manuscript and approved the final version of the manuscript.

\section{Funding sources:}

None available

The patient consent statement : A written consent has been obtained before submission and be made available to the publisher if requested.

Authorship statement: Ozaki A, Kaneda Y, and Senoo Y wrote the manuscript. The other authors made substantial contributions to the design of the study and critical revision of the manuscript.

Conflicts of interest statement : Ozaki A receives personal fees from MNES Inc, outside the submitted work. Tetsuya Tanimoto receives personal fees from MNES Inc, and Bionics co., ltd. outside the submitted work.

\section{References}

1. Syed A, Kumari G, Kapoor A, et al. Impact of COVID-19 On Breast Cancer Management: A Radiological Prespective from A Tertiary Centre. Eur J Breast Health . 2021;17(2):180-187.

2. Ozaki A, Sawano T, Saito H, Tanimoto T, Tsubokura M. Will initial consultation patterns among undiagnosed cancer patients be the same after this COVID-19 pandemic? Experiences from the 2011 triple disaster in Fukushima, Japan. J Glob Health .2020;10(2):020343. 
3. Khatcheressian JL, Wolff AC, Smith TJ, et al. American Society of Clinical Oncology 2006 update of the breast cancer follow-up and management guidelines in the adjuvant setting. J Clin Oncol.2006;24(31):50915097.

4. Stuart K, Brennan M, French J, Houssami N, Boyages J. Life after breast cancer. Aust Fam Physician $.2006 ; 35(4): 219-224$.

5. Ozaki A, Tachibana K, Ohtake T. Challenges and future directions in breast cancer care in Fukushima prefecture in Japan: correspondence to "A survey on the current status of clinical resources for diagnosis and treatment of breast cancer in rural hospitals of the Tohoku region in Japan". Breast Cancer .2021;28(5):11631164 .

6. Bokolo Anthony J. Use of Telemedicine and Virtual Care for Remote Treatment in Response to COVID-19 Pandemic. J Med Syst.2020;44(7):132.

7. Fouquet SD, Miranda AT. Asking the Right Questions-Human Factors Considerations for Telemedicine Design. Curr Allergy Asthma Rep. 2020;20(11):66.

8. Chen Z, Sun S, Zhao W, et al. The Impact of the Declaration of the State of Emergency on the Spread of COVID-19: A Modeling Analysis. Comput Math Methods Med.2021;2021:8873059.

9. Ekeland AG, Bowes A, Flottorp S. Effectiveness of telemedicine: a systematic review of reviews. Int $J$ Med Inform.2010;79(11):736-771. 